\title{
The prognostic value of neutrophil-lymphocyte ratio is superior to derived neutrophil- lymphocyte ratio in advanced gastric cancer treated with preoperative chemotherapy and sequential $\mathrm{R} 0$ resection: a 5-year follow-up
}

This article was published in the following Dove Press journal:

OncoTargets and Therapy

22 May 2017

Number of times this article has been viewed

Hailong Jin

Jianyi Sun

Kankai Zhu

Xiaosun Liu

Qing Zhang

Qianyun Shen

Yuan Gao

Jiren Yu

Department of Gastrointestinal Surgery, the First Affiliated Hospital, Medical College, Zhejiang University, Hangzhou, People's Republic of China

Correspondence: Jiren $\mathrm{Yu}$

Department of Gastrointestinal Surgery, the First Affiliated Hospital, Medical College, Zhejiang University, No 79, Qingchun Road, 3I 0003

Hangzhou, Zhejiang Province, People's

Republic of China

$\mathrm{Tel}+8657 \mid 87236147$

Fax +86 57| 87072577

Email yujr0909@zju.edu.cn
Aim: The role of neutrophil-lymphocyte ratio (NLR) and derived neutrophil-lymphocyte ratio (d-NLR) in outcome prediction is assessed in patients with advanced gastric cancer receiving preoperative chemotherapy in a 5-year follow-up cohort.

Patients and methods: Patients undergoing preoperative chemotherapy and sequential R0 resection for advanced gastric cancer were enrolled from July 2004 to November 2011. Wilcoxon matched-pairs signed-rank test was used to evaluate the change of peripheral blood parameters. Receiver operating curve was used to identify the optimal cutoff values of NLR and d-NLR. Survival function was analyzed using Kaplan-Meier curves and Cox proportional hazard model.

Results: Significant difference was found between baseline and post-chemotherapy blood parameters, including leukocytes, neutrophils, lymphocytes, NLR and d-NLR (all $P<0.05$ ). High baseline NLR group (NLR $\geq 2.230$ ) had a significant shorter recurrence-free survival (RFS) (hazard ratio $[\mathrm{HR}]=1.814,95 \%$ confidence interval $[95 \% \mathrm{CI}]: 1.112-2.960$, $P=0.015)$ and shorter overall survival (OS) (HR $=1.867,95 \%$ CI: $1.129-3.089, P=0.013$ ) than those of the low baseline NLR group (NLR <2.230). High baseline d-NLR group (d-NLR $\geq 1.885$ ) also had a shorter RFS (HR $=1.805,95 \%$ CI: $1.116-2.919, P=0.014)$ and shorter OS (HR $=1.783,95 \% \mathrm{CI}: 1.091-2.916, P=0.019)$ than those of the low baseline d-NLR group (d-NLR <1.885). However, post-chemotherapy NLR and d-NLR showed no prognostic significance on RFS and OS (all $P>0.05$ ). Multivariate analysis showed that higher baseline NLR but not d-NLR was identified as an independent factor associated with worse RFS (HR $=1.707,95 \%$ CI: $1.042-2.797, P=0.034$ ) and worse OS (HR $=1.758,95 \%$ CI: $1.058-2.919, P=0.029)$.

Conclusion: Baseline NLR and d-NLR may serve as convenient, easily measured prognostic indicators in advanced gastric cancer treated with preoperative chemotherapy and sequential R0 resection, especially to baseline NLR, which showed independent prognostic significance on RFS and OS, while post-chemotherapy NLR and d-NLR lost their usefulness due to the inhibition of bone marrow hematopoietic function. Patients with high baseline NLR and d-NLR values need multimodal therapy.

Keywords: advanced gastric cancer, preoperative chemotherapy, neutrophil, lymphocyte, prognosis 


\section{Introduction}

Gastric cancer is the fifth most common malignancy and the third leading cause of cancer death worldwide. Annually, nearly 1 million new cases are diagnosed, and $>700,000$ deaths are estimated to occur from this disease. ${ }^{1}$

Most of the newly diagnosed gastric cancer patients show advanced stage, where the early gastric cancer patients comprise $<10 \%$. Surgery is considered the main curative treatment for gastric cancer. However, the 5-year overall survival (OS) remains poor even after radical resection, especially when the primary tumor penetrates the serosa or invades the surrounding structure, ranging from $20 \%$ to $30 \%$. $^{2}$ Increased use of adjuvant and neo-adjuvant treatment regimens has greatly improved recurrence-free survival (RFS) and OS. ${ }^{3}$ Thus, comprehensive treatment regimens including surgery, chemotherapy and other strategies such as immunotherapy and target therapy are needed for advanced gastric cancer. Meanwhile, it is crucial to elucidate the biological mechanisms that contribute to tumor progression and identify prognostic indicators for risk stratification and subsequent improvement in the selection of gastric cancer patients for potential chemotherapy and radical resection.

There is increasing and consistent evidence that systemic inflammation is a key determinant of tumor progression in patients with cancer. ${ }^{4-6}$ Various markers of inflammation including cytokines, C-reactive protein (CRP), modified Glasgow prognostic score (mGPS), which combines CRP and albumin, and absolute leukocyte count and its components such as neutrophil, lymphocyte and their combined indexes such as NLR and d-NLR have been examined over the past decade in an attempt to refine stratification of patients to treatment and predict survival in a variety of solid and hematological cancers. ${ }^{7-9}$

The NLR is a simple, robust and convenient parameter of the systemic inflammation. Elevated baseline NLR was identified as an independent prognostic factor associated with dismal outcome in various types of cancers. ${ }^{10-12}$ Recently, the d-NLR was implemented ${ }^{9}$; they proposed a similar prognostic value of the d-NLR compared with the NLR in different solid cancer types, and its prognostic value was further proved in subsequent investigation in various types of cancers. ${ }^{13-16}$

However, the baseline NLR and posttreatment NLR have been found to be significant prognostic markers for survival prediction in patients who underwent systematic chemotherapy in several kinds of cancers, including gastric cancer. ${ }^{17-23}$ Nevertheless, evidence for the use of NLR and d-NLR as predictors of clinical outcome in patients with advanced gastric cancer treated with preoperative chemotherapy is still lacking, especially the data of long-term follow-up.
Therefore, the aim of this study was to evaluate the prognostic significance of baseline NLR and d-NLR and post-chemotherapy NLR and d-NLR in patients with gastric cancer at advanced stage in a cohort of 5-year follow-up.

\section{Patients and methods Study group}

A consecutive cohort of patients histopathologically confirmed with gastric cancer, treated with preoperative chemotherapy and followed by $\mathrm{R} 0$ resection (no residual) were retrospectively collected in our department from July 2004 to November 2011. Pathological tumor-node-metastasis (TNM) staging after preoperative chemotherapy was classified based on the 7 th edition of the American Joint Committee on Cancer. ${ }^{24}$ The inclusion criteria were as follows: 1) advanced stage based on computed tomography (CT) and endoscopy; 2) an age of 18-80 years; 3) Eastern Cooperative Oncology Group (ECOG) performance status of $0-2$; 4) R0 resection and 5) adequate blood count (white blood cell [WBC] count $>4 \times 10^{9} / \mathrm{L}$, platelets $\left.>100 \times 10^{9} / \mathrm{L}\right)$, creatinine clearance $>60 \mathrm{~mL} / \mathrm{min}$ and adequate hepatic function (bilirubin $<1.25 \times$ the upper limit of normal, AST/ ALT $<2.5 \times$ the upper limit of normal). The exclusion criteria included 1) distant metastases, 2) paraaortic lymph node metastasis, 3) prior chemotherapy or radiotherapy, 4) prior gastrectomy due to any reason, 5) history of another malignancy except cured basal cell carcinoma of skin and cured carcinoma in situ of uterine cervix, 6) presence of hematological disorders, acute inflammatory or autoimmune diseases or prior steroid treatment, and 7) blood transfusion within the last 3 months.

The study was approved by the ethical committees of the First Affiliated Hospital of Zhejiang University, School of Medicine, Hangzhou, People's Republic of China. As this is a retrospective nonintervention study, the institutional review board waived the need for written informed consent from the participants. Patient data will be kept confidentially.

\section{Treatment}

Preoperative chemotherapy regimens were as follows: 30 patients received oxaliplatin plus capecitabine (XELOX), 48 patients received oxaliplatin plus 5-fluorouracil (5-Fu) plus leucovorin (FOLFOX) and 37 patients received oxaliplatin plus S-1 (SOX). Another four patients received chemotherapy regimens as follows: EOF (which consisted of epirubicin plus oxaliplatin plus 5-Fu) for two patients, EOX (which consisted of epirubicin plus oxaliplatin plus capecitabine) for one patient and PS (which consisted of paclitaxel 
plus S-1) for one patient. If the disease progressed during the period of chemotherapy, patients received paclitaxelbased second-line chemotherapy regimens. Surgery was carried out within 2 weeks after the completion of last cycle of preoperative chemotherapy. Total distal gastrectomy or combined resection with D2 or D2+ lymphadenectomy was performed, depending on the location and extent of the primary tumor. Postoperative chemotherapy was continued within 4-6 weeks after surgery.

\section{Blood sample analysis}

Peripheral blood was obtained within 1 week of commencement of the first cycle of chemotherapy and within 1 week of resection. Venous blood samples were drawn into tubes containing ethylenediamine tetra-acetic acid (EDTA) for the measurement of hematological parameters, including leukocytes, neutrophils and lymphocytes. The NLR was calculated as absolute neutrophil count divided by the absolute lymphocyte count. The d-NLR was calculated as neutrophil count divided by leukocyte count minus neutrophil count.

\section{Follow-up}

All patients who completed the abovementioned treatment were followed up every 3-6 months for $1-2$ years, 6-12 months for 3-5 years, and annually for $>5$ years. The follow-up projects included blood routine test (BRT), biochemistry profile, tumor marker, endoscopy and radiologic imaging examinations, such as abdominal CT scan with intravenous (IV) contrast, ultrasonography and positron emission tomography plus computed tomography (PET-CT), emission computed tomography (ECT) if clinical indicated.

\section{Statistical analysis}

The medical record for each patient was reviewed independently by two physicians (HLJ and JYS) for clinicopathological characteristics. Comparison of peripheral blood parameters before and after preoperative chemotherapy was assessed by the Wilcoxon matched-pairs signed-rank test. The follow-up period commenced at the start of preoperative chemotherapy with a censor date of February 2017. RFS was defined as the time from the date of initiation of preoperative chemotherapy to the date of demonstration of recurrent disease, confirmed radiologically or histologically, or to the date of death or last contract. OS was defined as the time from date of initiation of preoperative chemotherapy to the date of death due to any cause, censoring patients who were alive. Receiver operating curve (ROC) was used to identify the optimal cutoff values of NLR and d-NLR.
Survival function was analyzed using Kaplan-Meier curves and compared by the log-rank test. Cox proportional hazard model was used to determine independent prognostic factors of clinicopathological variables.

All statistical analyses were performed using the SPSS software version 19.0 (SPSS Inc., Chicago, IL, USA). A twosided $P<0.05$ was considered statistically significant.

\section{Results}

\section{Patient demographics and outcomes}

A total of 119 advanced gastric cancer patients were eligible for this study; 87 were males and 32 were females. The median age was 59 years (range: $34-78$ years). The median number of preoperative chemotherapy cycles was 3 (range: 1-7). During the period of preoperative chemotherapy, four (3.4\%) patients received paclitaxel-based second-line chemotherapy regimens due to disease progression. All 119 patients underwent R0 resection with D2 or D2+ lymphadenectomy; among them, subtotal gastrectomy was performed for $56(47.1 \%)$ patients and total gastrectomy was performed for 55 (46.2\%) patients. Eight (6.7\%) patients underwent combined resection, with distal pancreatectomy plus splenectomy for three, splenectomy for four, and partial transverse colon for one, respectively. Pathological TNM classifications after preoperative chemotherapy were as follows: eight patients showed no residual tumor in the resection specimens, ten patients had stage I disease, 27 patients had stage II disease, and 72 had stage III disease (Table 1).

Of 119 patients, the median follow-up time was 84 months; all patients fulfilled the 5-year follow-up. A total of $67(56.3 \%)$ patients showed obvious recurrence that was confirmed radiologically or histologically, while 64 (53.8\%) patients died during the follow-up period. The median RFS was 46 months (range: 5-134 months) and median OS was 61 months (range: 8-134 months). The 1-year, 3-year, and 5 -year survival rates were $85.7 \%, 55.5 \%$, and $44.5 \%$ for RFS and $95.0 \%, 61.3 \%$, and $50.4 \%$ for OS, respectively (Figure 1A and B).

\section{Baseline and post-chemotherapy blood sample parameters}

The median values for baseline absolute leukocyte, neutrophil and lymphocyte counts were $5,800 / \mu \mathrm{L}, 3,600 / \mu \mathrm{L}$ and $1,500 / \mu \mathrm{L}$, respectively. The median values for post-chemotherapy absolute leukocyte, neutrophil, and lymphocyte counts were $4,600 / \mu \mathrm{L}, 2,500 / \mu \mathrm{L}$, and $1,300 / \mu \mathrm{L}$, respectively. Significant difference was found between baseline parameters and postchemotherapy parameters after completion of preoperative 
Table I Demographic and clinicopathological characteristics of II9 patients with advanced gastric cancer

\begin{tabular}{|c|c|}
\hline Parameters & Number (\%) \\
\hline \multicolumn{2}{|l|}{ Gender } \\
\hline Male & $87(73.1)$ \\
\hline Female & $32(26.9)$ \\
\hline \multicolumn{2}{|l|}{ Age, years } \\
\hline$<65$ & $83(69.7)$ \\
\hline$\geq 65$ & $36(30.3)$ \\
\hline \multicolumn{2}{|l|}{ ECOG } \\
\hline 0 & $49(4 \mid .2)$ \\
\hline I, 2 & $70(58.8)$ \\
\hline \multicolumn{2}{|l|}{ Chemotherapy regimen } \\
\hline XELOX & $30(25.2)$ \\
\hline FOLFOX & $48(40.3)$ \\
\hline sox & $37(31.1)$ \\
\hline Others ${ }^{\mathrm{a}}$ & $4(3.4)$ \\
\hline Cycle of chemotherapy, median (range) & $3(1-7)$ \\
\hline \multicolumn{2}{|l|}{$\begin{array}{l}\text { Primary tumor site } \\
\end{array}$} \\
\hline Upper & $20(16.8)$ \\
\hline Middle & $27(22.7)$ \\
\hline Lower & $60(50.4)$ \\
\hline More than two sites & $12(10.1)$ \\
\hline \multicolumn{2}{|l|}{ Tumor size, $\mathrm{cm}^{\mathrm{b}}$} \\
\hline$<4$ & $4 \mid(34.5)$ \\
\hline$\geq 4$ & $65(54.6)$ \\
\hline \multicolumn{2}{|l|}{ Gastrectomy } \\
\hline Total & $55(46.2)$ \\
\hline Subtotal & $56(47.1)$ \\
\hline Combined resection & $8(6.7)$ \\
\hline \multicolumn{2}{|l|}{ Lymphadenectomy } \\
\hline D2 & $84(70.6)$ \\
\hline D2+ & $35(29.4)$ \\
\hline \multicolumn{2}{|l|}{ Differentiation ${ }^{c}$} \\
\hline Well & $23(19.3)$ \\
\hline Poorly & $86(72.3)$ \\
\hline \multicolumn{2}{|l|}{ Pathological TNM classification ${ }^{d}$} \\
\hline \multicolumn{2}{|l|}{$*_{y p}$ T stage } \\
\hline T0 & $8(6.7)$ \\
\hline TI & $4(3.4)$ \\
\hline $\mathrm{T} 2$ & $19(16.0)$ \\
\hline T3 & $0(0)$ \\
\hline $\mathrm{T} 4$ & $86(72.3)$ \\
\hline \multicolumn{2}{|l|}{$*_{y p} \mathrm{~N}$ stage } \\
\hline No & $32(26.9)$ \\
\hline NIN2N3 & $87(73.1)$ \\
\hline \multicolumn{2}{|l|}{$*_{\text {yp TNM } \text { stage }^{f}}$} \\
\hline TONOMO & $3(2.5)$ \\
\hline $\mathrm{I}$ & $10(8.4)$ \\
\hline II & $27(22.7)$ \\
\hline III & $72(60.5)$ \\
\hline \multicolumn{2}{|l|}{ Postoperative chemotherapy ${ }^{8}$} \\
\hline Present & $100(84.0)$ \\
\hline Absent & $17(14.3)$ \\
\hline
\end{tabular}

Notes: ${ }^{\mathrm{a}}$ Others: including EOX, PS, EOF regimens. ' $T$ Tumor size: eight patients found no residual tumor in the resection specimens, while data of five patients were not available. 'Differentiation: well includes well- and moderately differentiated adenocarcinoma, poorly includes poorly differentiated adenocarcinoma, ring cell carcinoma, squamous carcinoma and mucinous adenocarcinoma. Eight patients found no residual tumor in the resection specimens, while data of two patients were not available. ${ }^{d}$ American Joint Committee on Cancer, 7th edition. ${ }^{24}$ eyp $T$ stage: data of two patients were not available because rare tumor cells were residual. fyp TNM stage: seven patients could not be classified according to the 7th TNM classification, three patients were TONIMO, two patients were TON2MO and

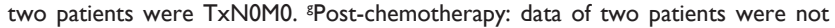
available. *Classification after preoperative chemotherapy.

Abbreviations: ECOG, Eastern Cooperative Oncology Group; TNM, tumor, node, metastasis; XELOX, oxaliplatin plus capecitabine; FOLFOX, oxaliplatin plus 5-fluorouracil plus leucovorin; SOX, oxaliplatin plus S-I. chemotherapy (for leukocyte, neutrophil, lymphocyte, Wilcoxon signed-rank test, all $P$-value $<0.05$; Table 2 ). The baseline NLR ranged from 0.38 to 10.67 , with a median value of 2.27, while the baseline d-NLR ranged from 0.29 to 5.33 , with a median value of 1.60 . The median values of post-chemotherapy NLR and d-NLR were 1.88 and 1.29, respectively. Similarly, significant difference was found between baseline NLR and d-NLR and post-chemotherapy NLR and d-NLR (for NLR: $P=0.004$; for d-NLR: $P<0.001$; Table 2). After preoperative chemotherapy, 74 patients showed a decreased value of NLR, while 44 patients showed an increased value and another one patient stays the same. For d-NLR, 80 patients showed a decreased value, while 36 patients showed an increased value and three patients stay the same.

\section{Correlation between clinicopathological features and survival (univariate analysis)}

The results of the individual Cox regression examining the association between each variable (including baseline and post-chemotherapy blood sample parameters) and RFS, as well as OS, are given in Table 3. For categorical variables, the hazard ratio (HR) represents the increased risk relative to the reference category. For continuous variables, the HR represents the increase in risk for each unit increase in value. In univariate analysis, poor differentiation, advanced $\mathrm{T}, \mathrm{N}$, and AJCC stages and higher baseline NLR and d-NLR were associated with higher risks of recurrence and mortality. The HR of RFS per unit increase of baseline NLR increased by 1.352 (95\% confidence interval [95\% CI]: 1.149-1.591, $P<0.001)$ and a similar HR of baseline d-NLR by 1.622 (95\% CI: 1.183-2.224, $P=0.003)$. Meanwhile, the HR of OS per unit increase of baseline NLR and d-NLR increased by 1.345 (95\% CI: 1.141-1.584, $P<0.001)$ and 1.601 (95\% CI: 1.170-2.192, $P=0.003)$, respectively. However, none of the post-chemotherapy parameters listed earlier showed prognostic significance on RFS and OS (all $P$-values $>0.05$ ).

\section{ROC analysis}

The optimal cutoff level was determined by applying ROC analysis. In estimating the 5-year risk of gastric cancer recurrence, the area under curve (AUC) was 0.591 (95\% CI: $0.489-0.693, P=0.09)$ for baseline NLR and $0.583(95 \%$ CI: $0.480-0.685, P=0.122$ ) for baseline d-NLR. In estimating the 5-year risk of mortality, the AUC was $0.634(95 \%$ CI: $0.534-0.735, P=0.011)$ for baseline NLR and 0.629 (95\% CI: $0.529-0.730, P=0.015$ ) for baseline d-NLR. A cutoff value of 2.230 was chosen as the optimal baseline NLR value for evaluation with RFS (sensitivity of $60.6 \%$ 

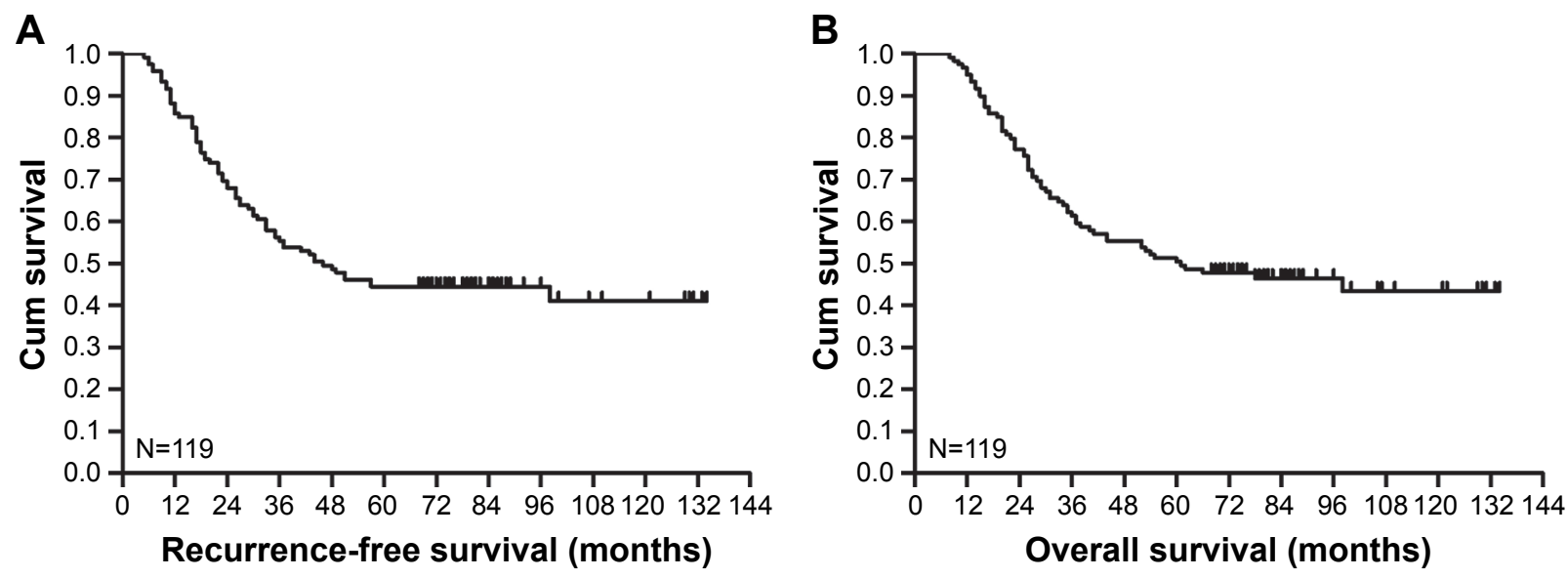

Figure I (A) RFS and (B) OS of II9 advanced gastric cancer patients treated with preoperative chemotherapy and R0 resection. Abbreviations: RFS, recurrence-free survival; OS, overall survival; Cum, cumulative.

and specificity of $56.6 \%$ ) and OS (sensitivity of $66.1 \%$ and specificity of $60.0 \%$ ). A cutoff value of 1.885 was chosen as the optimal baseline d-NLR value for RFS (sensitivity of $50.0 \%$ and specificity of $67.9 \%$ ) and OS (sensitivity of $54.2 \%$ and specificity of $70.0 \%$ ).

\section{Correlation between baseline NLR, baseline d-NLR and survival}

First, the total patients were divided into two groups (high group and low group) according to the cutoff value 2.230 of the baseline NLR, and 63 (52.9\%) patients had NLR $\geq 2.230$. The high baseline NLR group had a significant shorter RFS (1-year, 3-year, 5-year survival rates: 81.0\%, 44.4\%, $36.5 \%$ vs $94.6 \%, 67.9 \%, 53.6 \%$, respectively; $\mathrm{HR}=1.814$, 95\% CI: 1.112-2.960, $P=0.015$; Figure 2A) and shorter OS (1-year, 3-year, 5-year survival rates: 92.1\%, 50.8\%, 38.1\% vs $98.2 \%, 73.2 \%, 64.3 \%$, respectively; HR $=1.867,95 \%$ CI: 1.129-3.089, $P=0.013$; Figure 2B) than the low baseline NLR group. Next, we analyzed the association between baseline d-NLR (cutoff value of 1.885 , divided into high group and low group) and survival. A total of 50 (42.0\%) patients had $\mathrm{d}-\mathrm{NLR} \geq 1.885$. The high baseline $\mathrm{d}-\mathrm{NLR}$

Table 2 Comparison of peripheral blood tests parameters derived from samples of baseline and post-chemotherapy

\begin{tabular}{lll}
\hline Variables (N=I I 9) & $\mathbf{Z}$ & P-value \\
\hline cLeu & $-5.38 I$ & $<0.001$ \\
cNeu & -5.238 & $<0.001$ \\
cLym & -3.465 & 0.001 \\
cNLR & -2.894 & 0.004 \\
cd-NLR & -4.499 & $<0.001$
\end{tabular}

Note: Data were analyzed using Wilcoxon matched-pairs signed-rank test. Abbreviations: cLeu, comparison of leukocyte; cNeu, comparison of neutrophil; cLym, comparison of lymphocyte; cNLR, comparison of neutrophil-lymphocyte ratio; cd-NLR, comparison of derived neutrophil-lymphocyte ratio. group also had a shorter RFS (1-year, 3-year, 5-year survival rates: $78.0 \%, 42.0 \%, 34.0 \%$ vs $91.3 \%, 65.2 \%, 52.2 \%$, respectively; HR $=1.805,95 \%$ CI: $1.116-2.919, P=0.014$; Figure 2C) and shorter OS (1-year, 3-year, 5-year survival rates: $96.0 \%, 48.0 \%, 36.0 \%$ vs $94.2 \%, 71.0 \%, 60.9 \%$, respectively; $\mathrm{HR}=1.783,95 \% \mathrm{CI}: 1.091-2.916, P=0.019$; Figure 2D) than the low baseline d-NLR group.

\section{Independent risk factors in RFS and OS (multivariate analysis)}

The variables with values of $P<0.1$ in univariate analyses were included in the Cox proportional hazard multivariate models to identify independent risk factors. Baseline NLR and baseline d-NLR were dichotomized into high group and low group according to the cutoff values acquired from ROC. Because pathological stage derived from tumor invasion stage and lymph node involvements, only $\mathrm{T}$ stage and $\mathrm{N}$ stage were included in further multivariate models. Chemotherapy regimen was also included due to the potential influence caused by different biological behaviors of different drugs. The results identified that baseline higher NLR but not baseline higher d-NLR was an independent factor associated with worse RFS (HR $=1.683$, 95\% CI: $1.022-2.770, P=0.041)$ and worse $\mathrm{OS}(\mathrm{HR}=1.758$, 95\% CI: $1.058-2.919, P=0.029)$, as well as advanced T stage (RFS: HR $=2.345,95 \%$ CI: $1.065-5.164, P=0.034$; OS: HR $=2.728,95 \%$ CI: $1.169-6.368, P=0.020)$ and $\mathrm{N}$ stage (RFS: HR $=4.739,95 \%$ CI: $1.890-11.884, P=0.001$; OS: HR $=4.526,95 \%$ CI: $1.802-11.368, P=0.001$ ) (Table 4). Meanwhile, baseline NLR and baseline d-NLR were enrolled in the multivariate analysis as continuous variables. The results demonstrated that baseline higher NLR was also an independent factor associated with worse RFS (HR $=1.303$, 95\% CI: $1.110-1.530, P=0.001)$ and worse OS $(\mathrm{HR}=1.297$, 
Table 3 Cox proportional hazard model for RFS and OS among the 119 advanced gastric cancer patients (univariate analysis)

\begin{tabular}{|c|c|c|c|c|}
\hline \multirow[t]{2}{*}{ Parameters } & \multicolumn{2}{|l|}{ RFS } & \multicolumn{2}{|l|}{ OS } \\
\hline & HR (95\% Cl) & $P$-value & HR (95\% Cl) & $P$-value \\
\hline \multicolumn{5}{|l|}{ Gender (ref: male) } \\
\hline Female & $1.134(0.666-1.930)$ & 0.643 & $1.224(0.716-2.093)$ & 0.461 \\
\hline \multicolumn{5}{|l|}{ Age, years (ref: <65) } \\
\hline$\geq 65$ & $0.800(0.470-1.362)$ & 0.410 & $0.87 \mid(0.509-|.49|)$ & 0.615 \\
\hline \multicolumn{5}{|c|}{ Chemotherapy regimen (ref: FOLFOX) } \\
\hline XELOX & $1.210(0.673-2.177)$ & 0.524 & $1.204(0.657-2.205)$ & 0.548 \\
\hline sox & $0.822(0.454-1.486)$ & 0.516 & $0.889(0.487-1.623)$ & 0.703 \\
\hline Others & $0.929(0.22 I-3.902)$ & 0.920 & $0.899(0.213-3.788)$ & 0.884 \\
\hline \multicolumn{5}{|l|}{ Tumor size, cm (ref: <4) } \\
\hline$\geq 4$ & $1.623(0.964-2.732)$ & 0.068 & $1.533(0.906-2.597)$ & 0.112 \\
\hline \multicolumn{5}{|l|}{ Differentiation (ref: well) } \\
\hline Poorly & $2.217(1.096-4.482)$ & 0.027 & $2.001(0.988-4.056)$ & 0.054 \\
\hline \multicolumn{5}{|l|}{ y T stage (ref: T0-2) ${ }^{\mathrm{a}}$} \\
\hline T3-4 & $3.808(1.816-7.984)$ & $<0.001$ & $4.204(1.913-9.240)$ & $<0.001$ \\
\hline \multicolumn{5}{|l|}{ y $\mathrm{N}$ stage (ref: N0) } \\
\hline $\mathrm{NI}-3$ & $6.929(2.778-17.278)$ & $<0.001$ & $6.299(2.523-15.727)$ & $<0.001$ \\
\hline \multicolumn{5}{|l|}{ y TNM stage (ref: $0 / 1 / I I)^{b}$} \\
\hline III & $5.678(2.798-11.519)$ & $<0.001$ & $5.932(2.8 \mid 4-12.507)$ & $<0.001$ \\
\hline \multicolumn{5}{|c|}{ Post-operation chemotherapy (ref: present) } \\
\hline Absent & $1.459(0.778-2.733)$ & 0.239 & $1.645(0.875-3.094)$ & 0.122 \\
\hline \multicolumn{5}{|l|}{ Baseline } \\
\hline WBC counts & $1.089(0.944-1.256)$ & 0.242 & $1.080(0.934-1.249)$ & 0.300 \\
\hline Neutrophil counts & $1.152(0.974-1.363)$ & 0.098 & I.I45 (0.965-1.360) & 0.121 \\
\hline Lymphocyte counts & $0.749(0.44 I-1.270)$ & 0.283 & $0.736(0.427-1.267)$ & 0.269 \\
\hline NLR & 1.352 (I.I49-I.59I) & $<0.001$ & $1.345(I .|4|-\mid .584)$ & $<0.001$ \\
\hline d-NLR & $1.622(1.183-2.224)$ & 0.003 & $1.601(1.170-2.192)$ & 0.003 \\
\hline \multicolumn{5}{|l|}{ Post-chemotherapy } \\
\hline WBC counts & $1.056(0.9|8-1.2| 4)$ & 0.449 & I.08I (0.94I-I.242) & 0.271 \\
\hline Neutrophil counts & $0.977(0.9 \mid 5-1.043)$ & 0.485 & $0.98 \mid(0.924-1.042)$ & 0.538 \\
\hline Lymphocyte counts & $0.946(0.590-1.517)$ & 0.817 & $1.013(0.63 \mathrm{I}-1.627)$ & 0.958 \\
\hline NLR & 0.971 (0.900-I.048) & $0.45 I$ & $0.974(0.904-1.049)$ & 0.481 \\
\hline $\mathrm{d}-\mathrm{NLR}$ & $1.059(0.82 \mathrm{I}-1.365)$ & 0.661 & $1.096(0.852-1.4 \mathrm{II})$ & 0.475 \\
\hline
\end{tabular}

Notes: ${ }^{\mathrm{B}}$ Because of the low number of events for T0 and TI, stages T0, TI and T2 were combined. 'because of the low number of events for 0 and I, stages 0 , I and II were combined. For continuous variables, the HR gives the increase in risk for each unit increase in value. For categorical variables, the HR gives the increased risk relative to the reference category.

Abbreviations: FOLFOX, oxaliplatin plus 5-fluorouracil plus leucovorin; RFS, recurrence-free survival; OS, overall survival; HR, hazard ratio; Cl, confidence interval; TNM, tumor, node, metastasis; WBC, white blood cell; NLR, neutrophil-lymphocyte ratio; d-NLR, derived neutrophil-lymphocyte ratio; XELOX, oxaliplatin plus capecitabine; SOX, oxaliplatin plus S-I.

95\% CI: $1.104-1.524, P=0.002)$, as well as advanced T stage (RFS: $H R=2.373,95 \%$ CI: $1.079-5.221, P=0.032$; OS: HR $=2.767,95 \% \mathrm{CI}: 1.187-6.451, P=0.018)$ and N stage (RFS: $\mathrm{HR}=4.614,95 \% \mathrm{CI}: 1.842-11.559, P=0.001$; OS: $\mathrm{HR}=4.428$, 95\% CI: $1.764-11.115, P=0.002)$.

\section{Discussion}

During the past decade, a variety of inflammatory factors have been identified as prognostic indicators of cancer-related survival. Maltoni et $\mathrm{al}^{7}$ found that biological factors, such as leukocytosis, lymphocytopenia and CRP, reached level B evidence-based recommendations of prognostic correlation in advanced cancer patients. However, most of these studies have focused on the baseline parameters of inflammatory indicators, while the change during therapy of inflammatory factors, which may reflect the dynamic change of balance between host inflammatory response, immune response and the effect of therapy, is rarely studied..$^{10,11}$

Previously, there has been a report regarding the significant correlation between the NLR, NLR normalization after one cycle of chemotherapy and survival in advanced gastric cancer patients treated with FOLFOX chemotherapy regimen. ${ }^{20}$ The authors inferred that patients with an NLR value $<3$ had significantly higher median survival time than those with an NLR value of 3 or above (15.9 months vs 10.9 months). In addition, they found that patients with high NLR who reverted to normal after one cycle of chemotherapy had significant better median survival time than those patients whose NLR remained abnormal. However, the main defect of that study was that they ignored the vast importance of 
A

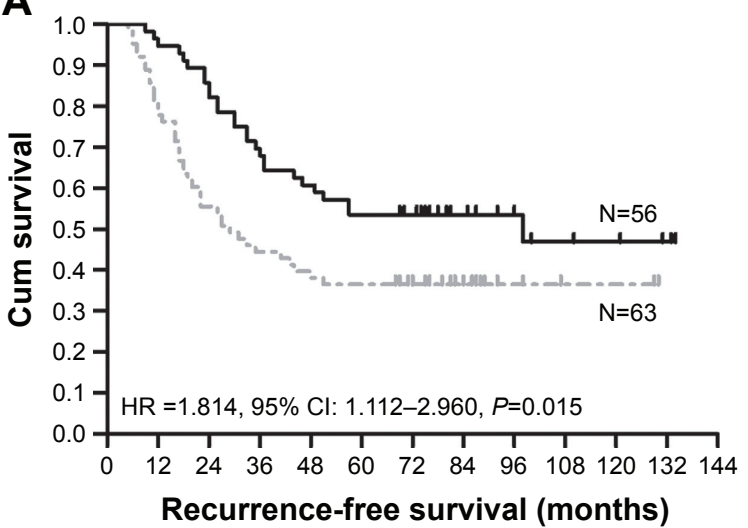

B

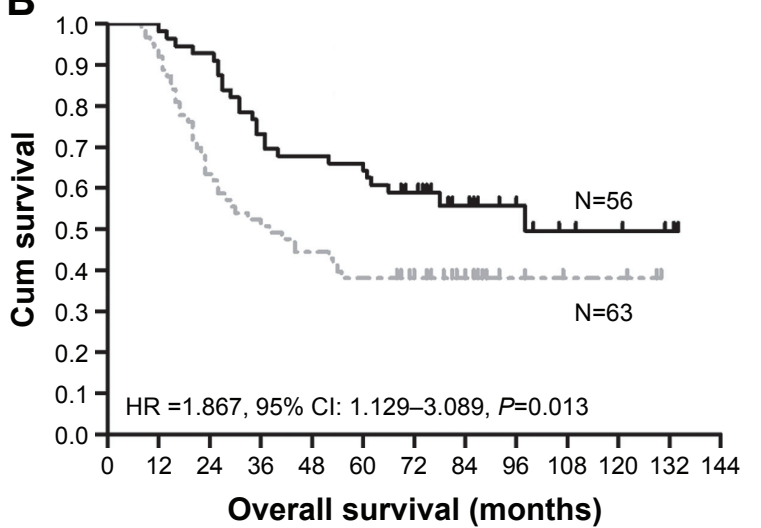

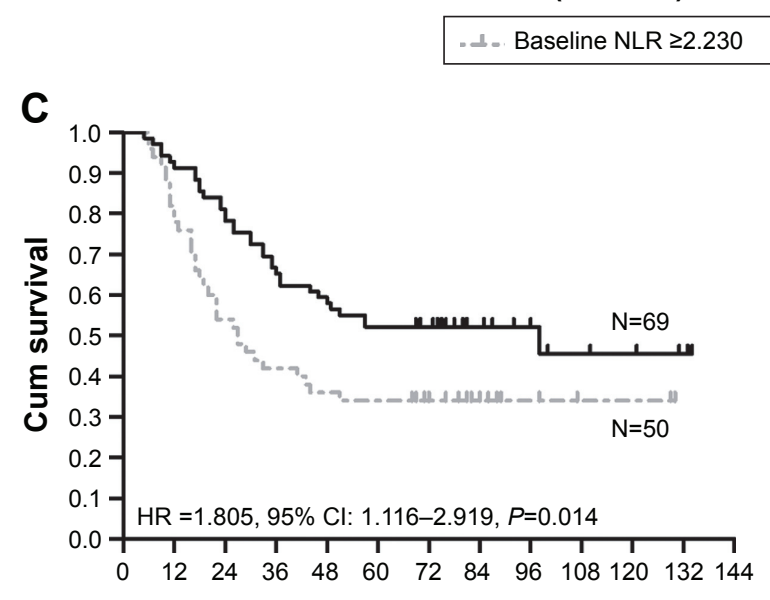

Recurrence-free survival (months) $\perp$ Baseline NLR $<2.230$

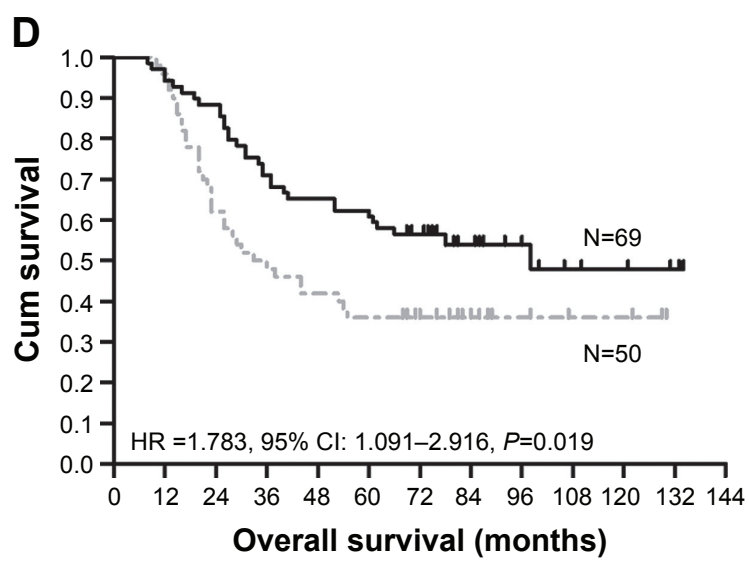

.ـ. Baseline d-NLR $\geq 1.885$ ـ Baseline d-NLR $<1.885$

Figure 2 Kaplan-Meier survival curves according to baseline NLR and baseline d-NLR.

Notes: Baseline NLR (cutoff value of 2.230) for (A) RFS and (B) OS; baseline d-NLR (cutoff value of I.885) for (C) RFS and (D) OS. P-values were determined using the log-rank test.

Abbreviations: NLR, neutrophil-lymphocyte ratio; d-NLR, derived neutrophil-lymphocyte ratio; RFS, recurrence-free survival; OS, overall survival; HR, hazard ratio; $\mathrm{Cl}$, confidence interval; cum, cumulative.

Table 4 Cox proportional hazard model for RFS and OS among the II 9 advanced gastric cancer patients (multivariate analysis)

\begin{tabular}{|c|c|c|c|c|}
\hline \multirow[t]{2}{*}{ Parameters } & \multicolumn{2}{|l|}{ RFS } & \multicolumn{2}{|l|}{ os } \\
\hline & HR (95\% Cl) & $P$-value & HR (95\% Cl) & $P$-value \\
\hline \multicolumn{5}{|c|}{ Chemotherapy regimen (ref: FOLFOX) } \\
\hline XELOX & $\mathrm{I} .444(0.784-2.66 \mathrm{I})$ & 0.239 & $1.436(0.773-2.668)$ & 0.253 \\
\hline sox & I.198 (0.639-2.248) & 0.573 & $1.152(0.6 \mid 4-2.162)$ & 0.659 \\
\hline Others & $1.246(0.283-5.487)$ & $0.77 I$ & $1.007(0.233-4.356)$ & 0.992 \\
\hline \multicolumn{5}{|l|}{ Tumor size, cm (ref: $<4$ ) } \\
\hline$\geq 4$ & $1.384(0.819-2.340)$ & 0.225 & & NA \\
\hline \multicolumn{5}{|l|}{ Differentiation (ref: well) } \\
\hline Poorly & I.448 (0.704-2.977) & 0.315 & $1.308(0.633-2.700)$ & 0.468 \\
\hline \multicolumn{5}{|l|}{ y T stage (ref: T0-2) } \\
\hline T3-4 & $2.345(1.065-5.164)$ & 0.034 & $2.728(1.169-6.368)$ & 0.020 \\
\hline \multicolumn{5}{|l|}{ y $\mathrm{N}$ stage (ref: N0) } \\
\hline $\mathrm{NI}-3$ & $4.739(1.890-11.884)$ & 0.001 & $4.526(1.802-\mid 11.368)$ & 0.001 \\
\hline \multicolumn{5}{|l|}{ Baseline parameters } \\
\hline NLR (ref: low group) & $1.683(1.022-2.770)$ & $0.04 I$ & 1.758 (1.058-2.919) & 0.029 \\
\hline d-NLR (ref: low group) & I.III (0.454-2.72I) & 0.817 & $0.897(0.363-2.217)$ & 0.814 \\
\hline
\end{tabular}

Note: Cox proportional multivariate hazards model was performed with the backward likelihood method.

Abbreviations: RFS, recurrence-free survival; OS, overall survival; HR, hazard ratio; Cl, confidence interval; NA, not applicable; NLR, neutrophil-lymphocyte ratio; d-NLR, derived neutrophil-lymphocyte ratio; XELOX, oxaliplatin plus capecitabine; FOLFOX, oxaliplatin plus 5-fluorouracil plus leucovorin; SOX, oxaliplatin plus S-I. 
surgery to survival, because they enrolled all patients when evaluating the correlation between NLR and survival, no matter whether patients had received operation, not to mention curative resection. Differently, in this study, we only enrolled advanced gastric cancer patients who had received preoperative chemotherapy with sequential R0 resection. Our results showed that peripheral blood parameters were significantly altered by chemotherapy, including leukocytes, neutrophils, lymphocytes, and their combination of NLR and d-NLR (Wilcoxon signed-rank test, all $P$-values $<0.05$ ). These results indicated that post-chemotherapy peripheral blood parameters might not be suitable for the use of prediction of survival due to the inhibition of bone marrow hematopoietic function caused by chemotherapeutics. To confirm this, individual Cox regression model was used for evaluating the association between each variable and survival. Patients with a high baseline NLR had a worse RFS (HR $=1.352,95 \%$ CI: 1.149-1.591, $P<0.001)$ and worse OS (HR $=1.345,95 \%$ CI: $1.141-1.584, P<0.001)$ than those with low NLR. However, the post-chemotherapy NLR showed no prognostic significance $(P=0.451$ for RFS and $P=0.481$ for OS). D-NLR was also evaluated in this study, whose prognostic significance was firstly validated by Proctor et $\mathrm{al}^{9}$ in patients with a variety of cancers. The results showed that patients with a high baseline d-NLR also had a worse RFS (HR $=1.622,95 \% \mathrm{CI}: 1.183-2.224, P=0.003$ ) and worse OS (HR $=1.601,95 \% \mathrm{CI}: 1.170-2.192, P=0.003)$ than those with low d-NLR, while post-chemotherapy d-NLR also showed no values in predicting survival $(P=0.661$ for RFS and $P=0.475$ for $\mathrm{OS}$ ).

In this study, ROC was applied to identify the optimal cutoff value of NLR and d-NLR. The high baseline NLR group (NLR $\geq 2.230$ ) had a significant shorter RFS $(\mathrm{HR}=1.814, P=0.015)$ and shorter $\mathrm{OS}(\mathrm{HR}=1.867, P=0.013)$ than the low baseline NLR group (NLR <2.230), as well as the d-NLR (high baseline d-NLR group [ $\geq 1.885]$ vs low baseline d-NLR group [ $<1.885]$, RFS: $\mathrm{HR}=1.805, P=0.014$; OS: $\mathrm{HR}=1.783, P=0.019)$. Multivariate analysis indicated that baseline NLR but not baseline d-NLR was an independent prognostic factor on both RFS and OS. This superiority perhaps due to the stability of NLR compared with absolute counts could be altered by various physiological and pathological factors. Based on the definition of d-NLR, the white cell count minus the neutrophil count was considered to be similar to the lymphocyte count, which ignored the importance of monocyte. As there is a relationship between the monocyte count and cancer, ${ }^{25,26}$ this association may explain the superiority of NLR to d-NLR.

The association between inflammatory indicators and poor survival in patients with various types of cancers has not been clearly defined until now. Indeed, there are several possible explanations for this. Relative neutrophilia increases the number of inflammatory markers that include vascular endothelial growth factor (VEGF), interleukin (IL)-18, and matrix metalloproteinases (MMPs) and antiapoptotic markers $(\mathrm{NF}-\kappa \mathrm{B})$ that support tumor growth and progression. ${ }^{27,28}$ On the other hand, lymphocytopenia represents a significant decline in the lymphocyte-mediated antitumor cellular immune response, demonstrated by the marked decrease in T4 helper and T8 suppressor lymphocytes. Furthermore, a number of studies have undertaken measurements of circulating cytokines together with the inflammatory factors. For example, Motomura et $\mathrm{al}^{29}$ showed that an elevated NLR was associated with an increase in IL-17 and peritumoral infiltration of tumor-associated macrophages (TAMs). Kantola et $\mathrm{al}^{30}$ reported that an elevated NLR was associated with elevated serum concentrations of IL-6, IL-7, IL-8, IL-12 and MCP-1 in colorectal carcinoma. Despite the increasing evidence that inflammatory cytokines play an important role in the tumorhost interaction, these links require further investigation.

To our knowledge, this is the first study to describe the use of NLR and d-NLR in advanced gastric cancer patients receiving preoperative chemotherapy and sequential $\mathrm{R} 0$ resection in terms of providing useful information regarding prognostication in a long-term follow-up. The major limitations of this study are the retrospective nature of the analysis and the single-center experience. We are unable to exclude the possibility that unequal distribution of unidentified clinicopathological variables and heterogeneous treatment regimens in our patient cohort that may have biased the results was observed. Although, apparently inferior to other measures of the systemic inflammatory factors, such as the mGPS (by Proctor), we could not analyze CRP or Glasgow prognostic score in this study, because CRP was not routinely measured in our institution. Additional, several tumor markers, such as carcinoembryonic antigen (CEA) and carbohydrate antigen (CA-199), were also not evaluated, because the missing data exceeded $10 \%$, which might bias the results. In conclusion, further prospective studies are needed to evaluate baseline and post-chemotherapy NLR, d-NLR and other biomarkers of inflammation for outcome prediction and therapeutic monitoring in patients with advanced gastric cancer receiving preoperative chemotherapy.

\section{Conclusion}

In a word, baseline NLR and d-NLR may serve as convenient, easily measured prognostic indicators in advanced gastric cancer treated with preoperative chemotherapy and sequential R0 resection, especially to baseline NLR, which 
showed independent prognostic significance on RFS and OS. While post-chemotherapy NLR and d-NLR lost their usefulness due to the inhibition of bone marrow hematopoietic function. Patients with high baseline NLR and d-NLR values need intensive therapy.

\section{Acknowledgments}

The authors thank Yingchun Ma in the Department of Gastrointestinal Surgery and laboratory physicians in the Clinical Laboratory, First Affiliated Hospital, Medical College, Zhejiang University, for blood sampling and testing. This study was supported by the grant of The Traditional Chinese Medicine (TCM) Science and Technology Project of Zhejiang Province (2014ZZ005) and the grant of The Major Science and Technology Project of Zhejiang Province (2014C03040-1).

\section{Author contributions}

All authors contributed toward data collection, analysis, drafting and revising the article and agree to be accountable for all aspects of this work.

\section{Disclosure}

The authors report no conflicts of interest in this work.

\section{References}

1. Ferlay J, Soerjomataram I, Dikshit R, et al. Cancer incidence and mortality worldwide: sources, methods and major patterns in GLOBOCAN 2012. Int J Cancer. 2015;136(5):E359-E386.

2. Isobe $\mathrm{Y}$, Nashimoto A, Akazawa K, et al. Gastric cancer treatment in Japan: 2008 annual report of the JGCA nationwide registry. Gastric Cancer. 2011;14(4):301-316.

3. Cunningham D, Allum WH, Stenning SP, et al. Perioperative chemotherapy versus surgery alone for resectable gastroesophageal cancer. N Engl J Med. 2006;355(1):11-20.

4. Balkwill F, Mantovani A. Inflammation and cancer: back to Virchow? Lancet. 2001;357(9255):539-545.

5. Coussens LM, Werb Z. Inflammation and cancer. Nature. 2002; 420(6917):860-867.

6. Hanahan D, Weinberg RA. Hallmarks of cancer: the next generation. Cell. 2011;144(5):646-674.

7. Maltoni M, Caraceni A, Brunelli C, et al. Prognostic factors in advanced cancer patients: evidence-based clinical recommendations - a study by the Steering Committee of the European Association for Palliative Care. J Clin Oncol. 2005;23(25):6240-6248.

8. Proctor MJ, Morrison DS, Talwar D, et al. A comparison of inflammation-based prognostic scores in patients with cancer. A Glasgow Inflammation Outcome Study. Eur J Cancer. 2011;47(17):2633-2641.

9. Proctor MJ, McMillan DC, Morrison DS, Fletcher CD, Horgan PG, Clarke SJ. A derived neutrophil to lymphocyte ratio predicts survival in patients with cancer. Br J Cancer. 2012;107(4):695-699.

10. Guthrie GJ, Charles KA, Roxburgh CS, Horgan PG, McMillan DC, Clarke SJ. The systemic inflammation-based neutrophil-lymphocyte ratio: experience in patients with cancer. Crit Rev Oncol Hematol. 2013; 88(1):218-230.
11. Paramanathan A, Saxena A, Morris DL. A systematic review and metaanalysis on the impact of pre-operative neutrophil lymphocyte ratio on long term outcomes after curative intent resection of solid tumours. Surg Oncol. 2014;23(1):31-39.

12. Pan QX, Su ZJ, Zhang JH, Wang CR, Ke SY. A comparison of the prognostic value of preoperative inflammation-based scores and TNM stage in patients with gastric cancer. Onco Targets Ther. 2015;8:1375-1385.

13. Absenger G, Szkandera J, Pichler M, et al. A derived neutrophil to lymphocyte ratio predicts clinical outcome in stage II and III colon cancer patients. Br J Cancer. 2013;109(2):395-400.

14. Szkandera J, Stotz M, Eisner F, et al. External validation of the derived neutrophil to lymphocyte ratio as a prognostic marker on a large cohort of pancreatic cancer patients. PLoS One. 2013;8(11):e78225.

15. Dirican A, Ekinci N, Avci A, et al. The effects of hematological parameters and tumor-infiltrating lymphocytes on prognosis in patients with gastric cancer. Cancer Biomark. 2013;13(1):11-20.

16. Dirican A, Kucukzeybek BB, Alacacioglu A, et al. Do the derived neutrophil to lymphocyte ratio and the neutrophil to lymphocyte ratio predict prognosis in breast cancer? Int J Clin Oncol. 2015;20(1):70-81.

17. Azab B, Bhatt VR, Phookan J, et al. Usefulness of the neutrophil-tolymphocyte ratio in predicting short- and long-term mortality in breast cancer patients. Ann Surg Oncol. 2012;19(1):217-224.

18. Chua W, Charles KA, Baracos VE, Clarke SJ. Neutrophil/lymphocyte ratio predicts chemotherapy outcomes in patients with advanced colorectal cancer. Br J Cancer. 2011;104(8):1288-1295.

19. Noble F, Hopkins J, Curtis N, et al. The role of systemic inflammatory and nutritional blood-borne markers in predicting response to neoadjuvant chemotherapy and survival in oesophagogastric cancer. Med Oncol. 2013;30(3):596.

20. Lee S, Oh SY, Kim SH, et al. Prognostic significance of neutrophil lymphocyte ratio and platelet lymphocyte ratio in advanced gastric cancer patients treated with FOLFOX chemotherapy. BMC Cancer. 2013;13:350.

21. Jin H, Zhang G, Liu X, et al. Blood neutrophil-lymphocyte ratio predicts survival for stage III-IV gastric cancer treated with neoadjuvant chemotherapy. World J Surg Oncol. 2013;11(112):1-10.

22. Luo G, Guo M, Liu Z, et al. Blood neutrophil-lymphocyte ratio predicts survival in patients with advanced pancreatic cancer treated with chemotherapy. Ann Surg Oncol. 2015;22(2):670-676.

23. Kao SC, Pavlakis N, Harvie R, et al. High blood neutrophil-to-lymphocyte ratio is an indicator of poor prognosis in malignant mesothelioma patients undergoing systemic therapy. Clin Cancer Res. 2010; 16(23):5805-5813.

24. Washington $\mathrm{K}$. 7th edition of the AJCC cancer staging manual: stomach. Ann Surg Oncol. 2010;17(12):3077-3079.

25. Sasaki A, Iwashita Y, Shibata K, Matsumoto T, Ohta M, Kitano S. Prognostic value of preoperative peripheral blood monocyte count in patients with hepatocellular carcinoma. Surgery. 2006;139(6):755-764.

26. Lee YY, Choi CH, Sung CO, et al. Prognostic value of pre-treatment circulating monocyte count in patients with cervical cancer: comparison with SCC-Ag level. Gynecol Oncol. 2012;124(1):92-97.

27. Jablonska E, Puzewska W, Grabowska Z, Jablonski J, Talarek L. VEGF, IL-18 and NO production by neutrophils and their serum levels in patients with oral cavity cancer. Cytokine. 2005;30(3):93-99.

28. Ardi VC, Kupriyanova TA, Deryugina EI, Quigley JP. Human neutrophils uniquely release TIMP-free MMP-9 to provide a potent catalytic stimulator of angiogenesis. Proc Natl Acad Sci U S A. 2007; 104(51):20262-20267.

29. Motomura T, Shirabe K, Mano Y, et al. Neutrophil-lymphocyte ratio reflects hepatocellular carcinoma recurrence after liver transplantation via inflammatory microenvironment. J Hepatol. 2013;58(1):58-64.

30. Kantola T, Klintrup K, Vayrynen JP, et al. Stage-dependent alterations of the serum cytokine pattern in colorectal carcinoma. Br J Cancer. 2012; 107(10):1729-1736 


\section{Publish your work in this journal}

OncoTargets and Therapy is an international, peer-reviewed, open access journal focusing on the pathological basis of all cancers, potential targets for therapy and treatment protocols employed to improve the management of cancer patients. The journal also focuses on the impact of management programs and new therapeutic agents and protocols on

patient perspectives such as quality of life, adherence and satisfaction. The manuscript management system is completely online and includes a very quick and fair peer-review system, which is all easy to use. Visit http://www.dovepress.com/testimonials.php to read real quotes from published authors.

Submit your manuscript here: http://www.dovepress.com/oncotargets-and-therapy-journal 